\title{
The prevalence of the Wernicke-Korsakoff syndrome in Sydney, Australia: a prospective necropsy study
}

\author{
CLIVE HARPER, * JULIAN GOLD, $†$ MICHAEL RODRIGUEZ, ${ }^{*}$ \\ MICHAEL PERDICES $\dagger$
}

From the Department of Pathology,* The University of Sydney, Sydney, NSW and Department of Anatomical Pathology, Royal Prince Alfred Hospital, Camperdown, NSW and AIDS Unit, Surry Hills, NSW, Australia.

SUMMARY In a prospective necropsy study, the prevalence of the Wernicke-Korsakoff syndrome (WKS) in Sydney, Australia was $2 \cdot 1 \%$ of adults over the age of 15 years. The population studied encompassed a wide spectrum of socio-economic and cultural backgrounds. Abuse of alcohol appeared to be the major predisposing factor to the development of the WKS in cases which were adequately documented. This high prevalence rate is in line with other clinical and pathological Australian studies and provides additional support for the idea of prevention of the WKS by the use of thiamin supplements in the Australian diet in flour, bread and perhaps alcoholic beverages.

There is still considerable interest in the prevalence of the Wernicke-Korsakoff Syndrome (WKS) in different population groups throughout the world. This disease is caused by thiamin deficiency but is seen most commonly in association with the abuse of alcohol.' There appears to be an unusually high prevalence in Australia based on both clinical and pathological studies. First admissions to Queensland hospitals with a diagnosis of WKS occurred at a rate of 65 per million population ${ }^{2}$ compared with 8 per million in New York ${ }^{3}$ and 16.9 in New Zealand. ${ }^{4}$ In a study of patients in mental hospitals in Queensland, Price and Theodoras determined that $15 \%$ had the WKS. ${ }^{2}$ Yellowlees noted an identical figure $(15 \%)$ in his study of numbers of patients with the WKS occupying licensed psychiatric hospital beds in South Australia.s From necropsy studies the highest prevalence figure in the world is from Western Australia $(2 \cdot 8 \%){ }^{6}$ Other figures include Cleveland, (Ohio): $2 \cdot 2 \%,{ }^{7}$ New York: $1 \cdot 7 \%{ }^{8}$ and Oslo: $0 \cdot 8 \% .^{9}$ The reason for this apparently high prevalence of the WKS in Australia is unclear but possible explanations include patterns of drinking (such as binge drinking), the lack of fortification of basic foods such as flour and

Address for reprint requests: Professor Clive Harper, Department of Pathology, The University of Sydney, Sydney, NSW 2006. Australia.

Received 27 September 1988.

Accepted 21 October 1988 bread by thiamin, and the fact that most Australian beer has effectively no thiamin content. There may well be other contributing factors.

Such a high prevalence of the WKS in Australia has wide ranging social, medical, financial and ethical $s$ implications. The collection of additional data is always warranted and may help in establishing future guidelines for the prevention and management of the WKS.

It may provide a useful baseline from which one could monitor any reduction in prevalence if preventative measures such as thiamin fortification of alcoholic beverages is introduced in Australia.

\section{Material and methods}

Cases for this study were collected prospectively from necropsies performed in the major hospitals and the City Morgue (Department of Forensic Medicine, New South Wales Department of Health) in a defined area of Sydney. This area included the Inner Metropolitan region of Sydney (as defined by the Department of Health of NSW) and the local government areas of South Sydney, Wollahra, Waverley and Randwick. Only cases whose residential address was within this study area and who were over the age of 15 years were included in the analysis. (Cases of the WKS are rare in children less than fifteen years of age). This area was selected because it contained a convenient population size $(662,000,1981$ Census) and encompassed a wide spectrum of socio-economic and cultural backgrounds. No attempt was made to preselect cases on the basis of a history 
of alcoholism. All necropsies performed on preset days of the week which fulfilled the residential and age criteria as discussed above were included in the study.

To address the question of seasonal variations in the incidence of the WKS cases were collected during two periods of three months (March to May and September to November inclusive).

Brains were taken at necropsy and fixed in $10 \%$ formalin. After adequate fixation they were examined macroscopically and the brain stem and cerebellum removed by sectioning the midbrain at the level of the inferior colliculus. The cerebral hemispheres were then sectioned in the coronal plane at 10 $\mathrm{mm}$ intervals ensuring that the mamillary bodies were not cut. The external appearances of mamillary bodies was noted-whether they were normal in size and colour. A normal mamillary body measures $4-6 \mathrm{~mm}$ in diameter and they are the same colour as other grey matter structures such as thalamus and basal ganglia. A block which incorporated the mamillary bodies was taken from every case. This block was embedded in paraffin wax and processed for routine histological examination. Three $10 \mu \mathrm{m}$ sections were cut from the anterior, mid and posterior parts of the mamillary bodies in each case and stained with haematoxylin and eosin. These were examined by one of us $(\mathrm{CH})$, looking for evidence of the acute, subacute or chronic changes. Of the 285 cases approximated half (140) were from the Forensic Department and half (145) were from the Hospitals. There were over twice as many males as females in the study and the ages ranged from 17 to 93 years. The mean age for the male and female groups were 64 and 65 years respectively. The sex and age distributions for the Hospital and Forensic cases were identical. Cases which were considered to show changes consistent with the diagnosis of the WKS were shown to an independent Neuropathologist for diagnostic confirmation.

\section{Results}

\section{Prevalence}

Six cases of WKS were identified out of the study group of 285 . This is a prevalence of $2 \cdot 1 \%$. Four of the cases died in hospital and the other two were from the Department of Forensic Medicine.

\section{Clinical details}

Of the six cases diagnosed as the WKS five were male and one was female. The patients were aged from 44 to 61 years. On reviewing the hospital records of the four patients who had died in hospital the following information was noted:-all were heavy drinkers and had cirrhosis of the liver. Three patients died with pneumonia and two of these had acute or chronic pancreatitis. The patient with acute pancreatitis had had severe anorexia with vomiting for 6 weeks prior to death and the microscopic changes in the mamillary bodies suggested that her Wernicke's encephalopathy was of recent onset. All four patients were noted to be vague, confused, drowsy and/or disorientated. However, only two of the cases had focal neurological signs (nystagmus) and in those cases a diagnosis of
Wernicke's encephalopathy (WE) was suspected. All four patients were given parenteral thiamin whilst in hospital.

\section{Pathology}

No macroscopic abnormalities of the mamillary bodies were noted, even in the six cases which were found to have WE after microscopic examination. Histologically, one case had acute WE, two had subacute WE, two had chronic WE and one had chronic WE with superimposed acute changes. Microscopic changes in each case were typical of WE as previously described. ${ }^{1}$ There was some variation in microscopic appearances at the three different levels of the mamillary bodies, anterior, mid and posterior regions. In one case only two of the three sections were easily diagnosed as WE. Had the third section been the only sample examined the diagnosis would have been missed.

\section{Discussion}

Although this is a relatively small necropsy study of the WKS it is of interest to note that the incidence of $2 \cdot 1 \%$ is similar to a previous larger study carried out in Western Australia (2.8\%). ${ }^{6}$ Moreover the study reported here was carried out in a prospective fashion with no attempt at selection of cases based on, for example, a history of alcoholism. Other studies have probably been biased in case selection as evidenced by the high prevalence (4.7\%) of the WKS in Coroner's (Forensic) necropsies in the Perth study. ${ }^{6}$ The incidence of the WKS in Coroner's cases in this study was only $1.7 \%$. It is not surprising that the incidence of the WKS was much higher in the hospital population as many authors have noted that alcohol associated disorders account for a high proportion of all hospital admissions. ${ }^{1213}$ This high prevalence of the WKS has also been noted in clinical studies in Sydney ${ }^{14}$ and in Queensland. ${ }^{15}$ In a recent hospital based surveilance programme by Gold and his colleagues, in the same geographic area used in this study, the number of cases of the WKS per 100,000 population per annum was 6.5. ${ }^{14}$ Although the WKS is caused by thiamin deficiency it occurs most commonly in association with alcoholism. ${ }^{1910}$ There are many reasons why patients who abuse alcohol might have thiamin deficiency. Diet is often poor and thiamin intake marginal. ${ }^{4}$ Alcohol interferes with active gastrointestinal transport ${ }^{16}$ and liver disease, a common accompaniment of alcohol abuse, leads to reduced body stores and impaired metabolism of thiamin. ${ }^{17}$

Clinical features exhibited by the four patients who died in hospital were typical in that it is the exception rather than the rule for patients to have the classical clinical triad of altered mental state, ataxia and eye 
signs (nystagmus and/or ophthalmoplegia). ${ }^{18}$ In a study of 131 cases of the WKS by the author and his colleagues only $16 \%$ cases had all three clinical signs, $28 \%$ had two signs, $37 \%$ had only one sign and $19 \%$ had no signs. ${ }^{18}$ The most common clinical observation, seen in $74 \%$ of cases, was a disorder of higher mental function: confusion, disorientation or drowsiness. This pattern is borne out by this small study. Of the four cases with adequate clinical information, all were vague, disorientated, confused or drowsy. Only two patients had other clinical signs-nystagmus - and both of these cases were suspected to have WE and were treated appropriately with parenteral thiamin. All four of these patients died from causes other than the WKS. Hospital records did not state whether or not there was any improvement in neurological status as a result of thiamin administration. It should be noted that patients have died as a result of acute WE despite apparently adequate doses of parenteral thiamin.' This raises important questions about thiamin metabolism in such patients and how they should be managed. To complicate the issue further, massive doses of thiamin have been shown, in experimental models (rabbits), to cause central apnoeic crises. ${ }^{19}$ Moreover one of the authors has personally studied a case of acute WE in a 38 year old man who was given massive doses of thiamin ( $4 \mathrm{gm}$ per day), and was found dead in bed one week after his admission. The only abnormality found at necropsy was acute WE. However, sudden unexplained death has been noted in 32 of 51 cases of WE. ' With regard to the incidence of cases of acute or subacute WE in relation to seasonal variations, there were too few cases in this study to permit comment. However, we have reviewed the date of death for our previously reported cases of acute and subacute WE. ${ }^{10}$ Fifteen of the 41 cases $(37 \%)$ died in the three months August to October. Other cases were scattered fairly evenly throughout the year. This suggests that there maybe a preponderance of cases of the WKS during the winter months. The incidence of characteristic microscopic changes in the mamillary bodies in the WKS has been almost $100 \%$ in most necropsy studies. ${ }^{81020}$ Thus it seems unlikely that a significant number of cases could have been missed in this study by limiting the histological examination to mamillary bodies. However, it is important to note that there was no macroscopic abnormality of the mamillary bodies in any of the six cases.

Several authors have noted previously that the diagnosis of WKS will be missed in about $25 \%$ of cases if brains are not examined microscopically. ${ }^{1020}$ Macroscopic changes of the mamillary bodies have recently been documented during life in cases of the WKS. Using magnetic resonance imaging Charness and $\mathrm{De} \mathrm{La} \mathrm{Paz}$ were able to determine the volume of the mamillary bodies. ${ }^{21}$ They showed that seven of nine patients with the WKS had significantly smaller mamillary bodies than 36 of 37 control cases. Thus this technique may provide assistance in making the clinical diagnosis of the WKS (at least in chronic cases).

In the most recent Queensland study Price and his colleagues have shown an apparent reduction in the numbers of cases of the WKS being admitted to psychiatric hospital since $1975-1976 . .^{22}$ However, as shown by this study a necropsy prevalence rate of $2 \cdot 1 \%$ is still alarmingly high and such data support the proponents of thiamin supplementation in Australian diets. Which foods should be fortified is another question. After examination of the diet of "problem drinkers" Price and his colleagues concluded that fortification of flour alone would be unlikely to provide sufficient thiamin for that group. ${ }^{22}$ Hence, fortification of alcoholic beverages may be a more realistic approach.

This work was supported by grants from the NH \& MRC and the Australian Associated Brewers. Technical assistance was provided by Mr D Harper and the manuscript was typed by Ms F Ferguson.

\section{References}

1 Harper C. Wernicke's encephalopathy: a more common disease than realised. A neuropathological study of 51 cases. $J$ Neurol Neurosurg Psychiatry 1979;42:226-31.

2 Price J, Theodoros MT. The supplementation of alcoholic beverages with thiamin: a necessary preventative measure in Queensland? Aust NZ J Psychiatry 1979;13:315-20.

3 Centrewall BS, Criqui MH. Prevention of the Wernicke-Korsakoff syndrome. N Engl J Med 1978;299:285-9.

4 Wood B, Breen KL. Clinical thiamin deficiency in Australia: the size of the problem and approaches to prevention. Med J Aust 1980;1:461-4.

5 Yellowlees PM. Thiamin deficiency and prevention of the Wernicke-Korsakoff syndrome. A major public health problem. Med J Aust 1986;745:216-9.

6 Harper C. Wernicke's encephalopathy in Western Australia-a common preventable disease. Aust Alcohol Drug Rev 1983;2: 71-3.

7 Victor M, Laureno R. Neurological complications of alcohol abuse: epidemiological aspects. In: Schoenberg B, ed. Advances in Neurology Vol 19, New York: Raven Press, 1978;603-17.

8 Cravioto H, Korein J, Silberman J. Wernicke's encephalopathy: a clinical \& pathological study of 28 autopsied cases. Arch Neurol 1961;4:510-9.

9 Torvik A, Lindboe CF, Rodge S. Brain lesions in alcoholics: a neuropathological study with clinical correlations. J Neurol Sci 1982;56:233-48.

10 Harper CG. The incidence of Wernicke's encephalopathy in Australia-A neuropathological study of 131 cases. $J$ Neurol Neurosurg Psychiatry 1983;46:593-8.

11 Harper CG. Sudden, unexpected death and Wernicke's encephalopathy. A complication of prolonged intravenous feeding. Aust NZ J Med 1980;10:230-5.

12 Williams AT, Harding Burns F, Morey S. Prevalence of alcohol- 
ism in a Sydney teaching hospital. Med J Aust 1978;2:608-11.

13 Jarman CMB, Kellett JM. Alcoholism in the general hospital. $B r$ Med J 1979;2:469-72.

14 Gold J, Perdices M, Lardner K, et al. The Wernicke-Korsakoff Project. Final Report. NHMRC. Canberra: AGPS, 1985.

15 Price J, Kerr R. Some observations on the Wernicke-Korsakoff syndrome in Australia. Br J Addict 1985;80:69-76.

16 Hoyumps AM. Mechanisms of thiamin deficiency in chronic alcoholism. Am J Clin Nutr 1980;3:2750-61.

17 Breen KN, Buttigieg R, Iossifidis S, et al. Jejunal uptake of thiamin hydrochloride in man: influence of alcoholism and alcohol. Am J Clin Nutr 1985;42:121-6.

18 Harper CG, Giles M, Finlay-Jones R. Clinical signs in the
Wernicke Korsakoff complex: a retrospective analysis of 131 cases diagnosed at necropsy. J Neurol Neurosurg Psychiatry 1986;49:341-5.

19 Haley TJ, Flesher AM. A toxicity study of thiamin hydrochloride. Science 1946;104:567-8.

20 Victor M, Adams RD, Collins GH. The Wernicke-Korsakoff Syndrome. Philadelphia: F A Davis, 1971.

21 Charness ME, De La Paz RL. Mamillary body atrophy in Wernicke's encephalopathy: antemortem identification using magnetic resonance imaging. Ann Neurol 1987;22:595-600.

22 Price J, Kerr R, Hicks M, Nixon PF. The Wernicke-Korsakoff syndrome: a reappraisal in Queensland with special reference to prevention. Med J Aust 1987;147:561-5. 